\title{
Molecular Oxygen in the Nearest QSO Mrk 231
}

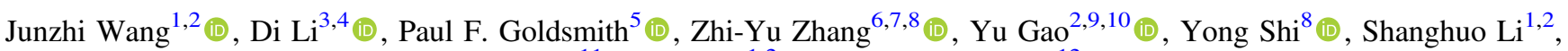 \\ Min Fang ${ }^{11}$ (D), Juan $\mathrm{Li}^{1,2}$, and Jiangshui Zhang ${ }^{12}$ (D) \\ ${ }^{1}$ Shanghai Astronomical Observatory, Chinese Academy of Sciences, 80 Nandan Road, Shanghai 200030 People’s Republic of China; jzwang@ @hao.ac.cn \\ ${ }^{2}$ Key Laboratory of Radio Astronomy, Chinese Academy of Sciences, Nanjing 210008, People's Republic of China \\ ${ }^{3}$ CAS Key Laboratory of FAST, National Astronomical Observatories, Chinese Academy of Sciences, Beijing 100012, People's Republic of China \\ ${ }^{4}$ University of Chinese Academy of Sciences, Beijing 100049, People's Republic of China \\ 5 Jet Propulsion Laboratory, California Institute of Technology, 4800 Oak Grove Drive, Pasadena, CA 91109, USA \\ ${ }^{6}$ Institute for Astronomy, University of Edinburgh, Royal Observatory, Blackford Hill, Edinburgh EH9 3HJ, UK \\ ${ }^{7}$ ESO, Karl Schwarzschild Strasse 2, D-85748 Garching, Munich, Germany \\ ${ }^{8}$ School of Astronomy and Space Science, Nanjing University, Nanjing 210093, People's Republic of China \\ ${ }^{9}$ Purple Mountain Observatory, Chinese Academy of Sciences, 2 West Beijing Road, Nanjing 210008, People's Republic of China \\ ${ }^{10}$ Department of Astronomy, Xiamen University, Xiamen, Fujian 361005, People's Republic of China \\ ${ }^{11}$ Department of Astronomy, University of Arizona, 933 North Cherry Avenue, Tucson, AZ 85721, USA \\ ${ }^{12}$ Center For Astrophysics, Guangzhou University, Guangzhou 510006, People's Republic of China \\ Received 2019 August 15; revised 2019 November 30; accepted 2019 December 10; published 2020 January 30
}

\begin{abstract}
We report the detection of an emission feature at the $12 \sigma$ level with FWHM line width of about $450 \mathrm{~km} \mathrm{~s}^{-1}$ toward the nearest quasi-stellar object, QSO Mrk 231. Based on observations with the IRAM $30 \mathrm{~m}$ telescope and the Northern Extended Millimeter Array Interferometer, the $1_{1}-1_{0}$ transition of molecular oxygen is the likely origin of the line with rest frequency close to $118.75 \mathrm{GHz}$. The velocity of the $\mathrm{O}_{2}$ emission in Mrk 231 coincides with the red wing seen in $\mathrm{CO}$ emission, suggesting that it is associated with the outflowing molecular gas, located mainly at about $10 \mathrm{kpc}$ away from the central active galactic nucleus (AGN). This first detection of extragalactic molecular oxygen provides an ideal tool to study AGN-driven molecular outflows on dynamic timescales of tens of megayears. $\mathrm{O}_{2}$ may be a significant coolant for molecular gas in such regions affected by AGN-driven outflows. New astrochemical models are needed to explain the implied high molecular oxygen abundance in such regions several kiloparsecs away from the center of galaxies.
\end{abstract}

Unified Astronomy Thesaurus concepts: Interstellar medium (847); Quasars (1319)

\section{Introduction}

As the third most abundant element in the universe after hydrogen and helium, oxygen and its chemistry in dense interstellar clouds are important for understanding the properties of molecular gas (Hollenbach et al. 2009; Goldsmith et al. 2011). Due to the attenuation of Earth's atmosphere, it is impossible to observe $\mathrm{O}_{2}$ lines near their rest frequencies from the ground. Searches for $\mathrm{O}_{2}$ emission in the Milky Way have been carried out from orbital observatories including the Submillimeter Wave Astronomy Satellite (SWAS) (Goldsmith et al. 2000, 2002), Odin (Liseau \& $\mathrm{O}_{2}$ din Team 2005; Larsson et al. 2007), and Herschel (Goldsmith et al. 2011; Liseau et al. 2012). Based on observations with those space missions (SWAS, Odin, and Herschel), the $\left[\mathrm{O}_{2}\right] /\left[\mathrm{H}_{2}\right]$ abundance ratios in dense gas regions are more than two orders of magnitude lower than predictions from pure gas-phase chemical models (Langer \& Graedel 1989; Bergin et al. 1998), $\sim 10^{-5}-\sim 10^{-4}$. Because of its low abundance and weak line emission, $\mathrm{O}_{2}$ is believed not to be an important coolant of the gas in dense molecular clouds.

The $\mathrm{O}_{2}$ line in extragalactic sources is redshifted away from the attenuation of the Earth's atmosphere, and thus can be observed with ground-based millimeter facilities. However, none of the observations detected $\mathrm{O}_{2}$ emission in the galaxies observed (Liszt 1985; Goldsmith \& Young 1989; Combes et al. 1991; Frayer et al. 1998; Kanekar \& Meier 2015) in the past two decades, with the best $\left[\mathrm{O}_{2}\right] /\left[\mathrm{H}_{2}\right]$ upper limit being $1 \times 10^{-6}$ in NGC 6240 at the $1 \sigma$ level (Combes et al. 1991), while the nondetection of absorption features toward a foreground galaxy in front of the $\mathrm{BL}$
Lac object B0218+357 give an upper limit of $2 \times 10^{-7}$ at the $1 \sigma$ level (Combes et al. 1997). Although the freeze-out of oxygen carriers, particularly $\mathrm{H}_{2} \mathrm{O}$, onto dust grains is generally cited as the explanation for the lack of gas-phase $\mathrm{O}_{2}$, a comprehensive picture of oxygen chemistry in different interstellar environments is still missing.

The enhancement of $\mathrm{O}_{2}$ emission in Orion (Goldsmith et al. 2011; Chen et al. 2014) is best explained by recent passage of a shock, while the active galactic nucleus (AGN)-driven molecular outflow can produce continuous shocks in associated molecular clouds. Thus, Mrk 231 is a good choice to search for extragalactic $\mathrm{O}_{2}$ emission. At a distance of $172 \mathrm{Mpc}$ (Fischer et al. 2010), Mrk 231 is the nearest QSO and the most luminous ultraluminous infrared galaxy $\left(L_{\mathrm{IR}}>10^{12} L_{\odot}\right)$ in the local universe (Feruglio et al. 2010). It contains high velocity molecular outflows revealed by far-infrared $\mathrm{OH}$ absorption (Fischer et al. 2010), and millimeter emission of CO (Feruglio et al. 2010; Cicone et al. 2012) and is observed in dense gas tracers (Aalto et al. 2012). The molecular mass outflow rate in Mrk 231 is estimated to be $700 M_{\odot} \mathrm{yr}^{-1}$ based on CO $J=1-0$ observations, which is higher than the star formation rate of $\sim 200 M_{\odot} \mathrm{yr}^{-1}$ (Feruglio et al. 2010).

The $\mathrm{O}_{2} \quad N_{J}=1_{1}-1_{0}$ transition with rest frequency $118.750343 \mathrm{GHz}$, is free from contamination by known nearby lines (Sandqvist et al. 2008). This spectral line, which can be observed toward local galaxies with redshift greater than $\sim 0.025$ using ground-based millimeter-wavelength facilities, is the best choice for a search for extragalactic molecular oxygen among $\mathrm{O}_{2}$ transitions. It was also the choice for the previous searches for the extragalactic $\mathrm{O}_{2}$ emission referred to above. 
In this paper, we describe the observations and data reduction in Section 2, present the main results in Section 3, discuss the results in Section 4, and give a brief summary and discuss future prospects in Section 5.

\section{Observations and Data Reduction}

\subsection{Observation with IRAM $30 \mathrm{~m}$ Telescope and Data Reduction}

The observations were carried out from 2015 August 20 to 23 , with the IRAM $30 \mathrm{~m}$ telescope in good weather conditions with precipitable water vapor less than $4 \mathrm{~mm}$ (Project id: 068-15, PI: Junzhi Wang). The Eight MIxer Receiver (EMIR) with dual polarizations, fast Fourier transform spectrometer backend, and standard wobbler switching mode at $0.5 \mathrm{~Hz}$ with $\pm 120^{\prime \prime}$ offset beam throw, were used toward Mrk 231 pointing at R.A. $=12: 56: 14.2 \mathrm{decl} .=+56: 52: 25.0$ (J2000). The beam size of the IRAM $30 \mathrm{~m}$ telescope at the observing frequency $(\sim 114 \mathrm{GHz})$ is about $23^{\prime \prime}$. In order to verify that the signal is from the sky rather than being radio-frequency interference at the IF frequency or from the backend, four local oscillator tuning setups were used during the observations, which are 103.77, 103.87, 103.57, and 103.07 GHz for August 20-23, respectively. We dumped the data every 1.7 minutes as one scan, and did one calibration observation every six scans. The CLASS package GILDAS ${ }^{13}$ was used for data reduction. During the data reduction, we checked the spectrum of each scan and dropped scans with bad baseline and/or hot spots in some channels, which comprised about $5 \%$ of the total data. We combined the data from all four days to obtain the final spectrum weighted by the rms of each individual scan. The rms of the final spectrum is $0.38 \mathrm{mK}$ in $T_{m b}$ after smoothing to the velocity resolution of $98.7 \mathrm{~km} \mathrm{~s}^{-1}$, while the total effective observing time (on+off) is 408 minutes.

\subsection{Observations with NOrthern Extended Millimeter Array (NOEMA) and Data Reduction}

The NOEMA observations were carried out on 2017 December 1 and 3 (Project id: d17ae001, PI: Junzhi Wang) under good weather conditions with the same pointing center as that of IRAM $30 \mathrm{~m}$ observation in 2015 . The frequency coverage of the upper sideband is from about 108.2 to $116.2 \mathrm{GHz}$, which covers the redshifted $\mathrm{CN} 1-0, \mathrm{CO} 1-0, \mathrm{O}_{2}$, and $\mathrm{HC}_{3} \mathrm{~N} J=13-12$ lines. The original frequency resolution was $2 \mathrm{MHz}$, which corresponds to $5.26 \mathrm{~km} \mathrm{~s}^{-1}$ at $114 \mathrm{GHz}$. On December 1, 3C84 was used as flux and bandpass calibrator, while $1300+580$ and $1150+497$ were used as phase calibrators, with seven antennae in the array and a total of $2.6 \mathrm{hr}$ time on Mrk 231. There were two observing blocks on December 3 using 3C273 as flux and bandpass calibrator while the phase calibrators were again $1300+580$ and $1150+497$. One block was done with eight antennae in the array and a total of $0.8 \mathrm{hr}$ on Mrk 231, while the other was done with nine antennae in the array and a total of $3.8 \mathrm{hr}$ on Mrk 231.

After accounting for bandpass, flux, phase, and amplitude calibrations for the visibility data with "CLIC" in GILDAS, we carried out imaging and deconvolving with both "MAPPING" in GILDAS and also CASA. The final results from both software packages are consistent. The images and spectra presented in this paper were done with CASA after smoothing to $10 \mathrm{MHz}$ frequency resolution corresponding to a velocity

\footnotetext{
${ }^{13}$ http://www.iram.fr/IRAMFR/GILDAS
}

resolution of $26.3 \mathrm{~km} \mathrm{~s}^{-1}$, and giving a slightly noncircular beam of $3.63^{\prime \prime} \times 3.32^{\prime \prime}$ with $\mathrm{PA}=-65^{\circ}$.

\section{Results}

\subsection{Detection of $\mathrm{O}_{2} \mathrm{~N}_{\mathrm{J}}=1_{1}-1_{0}$ Emission with the IRAM $30 \mathrm{~m}$ Telescope}

An emission feature was detected at about the $12 \sigma$ level, which we identify as $\mathrm{O}_{2} N_{J}=1_{1}-1_{0}$ emission with similar velocity range to that of the red wing of outflowing gas observed in $\mathrm{CO} J=1-0$. The velocity range of this feature is about $150-650 \mathrm{~km} \mathrm{~s}^{-1}$ (see Figure 1). The frequency resolution of the spectrum shown in Figure 1 is $37.5 \mathrm{MHz}$, which corresponds to $98.7 \mathrm{~km} \mathrm{~s}^{-1}$ at $113.945 \mathrm{GHz}$ for the $\mathrm{O}_{2}$ $N_{J}=1_{1}-1_{0}$ line with $z=0.04217$ (Bryant \& Scoville 1996; Carilli et al. 1998). This redshift corresponds to $12,642 \mathrm{~km} \mathrm{~s}^{-1}$ as the optically defined velocity for this galaxy from NASA/ IPAC Extragalactic Database (http://ned.ipac.caltech.edu).

The velocity-integrated flux of $\mathrm{O}_{2} N_{J}=1_{1}-1_{0}$ emission is $0.88 \pm 0.07 \mathrm{~K} \mathrm{~km} \mathrm{~s}^{-1}$, which corresponds to $4.4 \pm 0.35 \mathrm{Jy} \mathrm{km} \mathrm{s}^{-1}$ with the conversion factor of 5.0 from $\mathrm{K}$ to $\mathrm{Jy}$. The error of $0.07 \mathrm{~K} \mathrm{~km} \mathrm{~s}^{-1}$ is from single component Gaussian fitting for the spectrum, while there will be about $20 \%$ more uncertainties due to absolute flux calibration which should be considered if comparing with different observations. The flux of detected $\mathrm{O}_{2}$ emission in Mrk 231 is comparable to that of the red wing of $\mathrm{CO} 1-0$ previously detected (Cicone et al. 2012), which is $2.32 \pm$ $0.17 \mathrm{Jy} \mathrm{km} \mathrm{s}^{-1}$. Our CO $J=1-0$ data obtained simultaneously with the $\mathrm{O}_{2}$ in our IRAM $30 \mathrm{~m}$ observations provided similar results for the red wing of $\mathrm{CO} 1-0$, with similar flux level and velocity range (see Figure 1 ). In addition to $\mathrm{O}_{2} N_{J}=1_{1}-1_{0}$ line, we also detect $\mathrm{HC}_{3} \mathrm{~N} J=13-12$ line, at a significance of $8 \sigma$. This line is at $\sim 1200 \mathrm{~km} \mathrm{~s}^{-1}$ in the figures as the $\mathrm{O}_{2} N_{J}=1_{1}-1_{0}$ frequency corrected central velocity of the galaxy was used as reference frequency. The data are shown at higher velocity resolution and wider velocity coverage in Figure 2.

Since the on-source times on August 20 and 21 are about half of those on August 22 and August 23, and the system temperatures on August 20 and 21 are higher than those on August 22 and 23, most of which are lower than $300 \mathrm{~K}$, the main contributions to the final spectrum are from the data on August 22 and 23. We present the data of August 22 and 23 as the two spectra in Figure 3. Even though the noise levels of the two spectra are much higher than the 4 day combined spectrum, the two emission features, especially the one in the range $\sim 100-800 \mathrm{~km} \mathrm{~s}^{-1}$, can be seen in the individual spectra shown in Figure 3. On the other hand, due to very little on-source time in August 20 and 21 without any emission signature, the spectra are not shown here.

\subsection{Emission Features Confirmed with the NOEMA Interferometer}

The single-dish detections of $\mathrm{O}_{2} N_{J}=1_{1}-1_{0}$ and $\mathrm{HC}_{3} \mathrm{~N}$ $J=13-12$ emission in Mrk 231 were confirmed with observations using NOEMA. $\mathrm{O}_{2} N_{J}=1_{1}-1_{0}$ emission detected with NOEMA extends to $\sim 15^{\prime \prime}\left(1^{\prime \prime}=0.87 \mathrm{kpc}\right.$ for Mrk 231) from the center of the galaxy (see Figure 4). After the inclination correction with the average value of $\cos (\theta)$ for random sample, the physical projection will be $15 \times 0.87 \times \frac{\pi}{2}=20.5 \mathrm{kpc}$. However, since the real inclination is unknown, the distances to center in the discussion part are without such correction. The total flux of the identified emission features observed with NOEMA (see Table 1) 


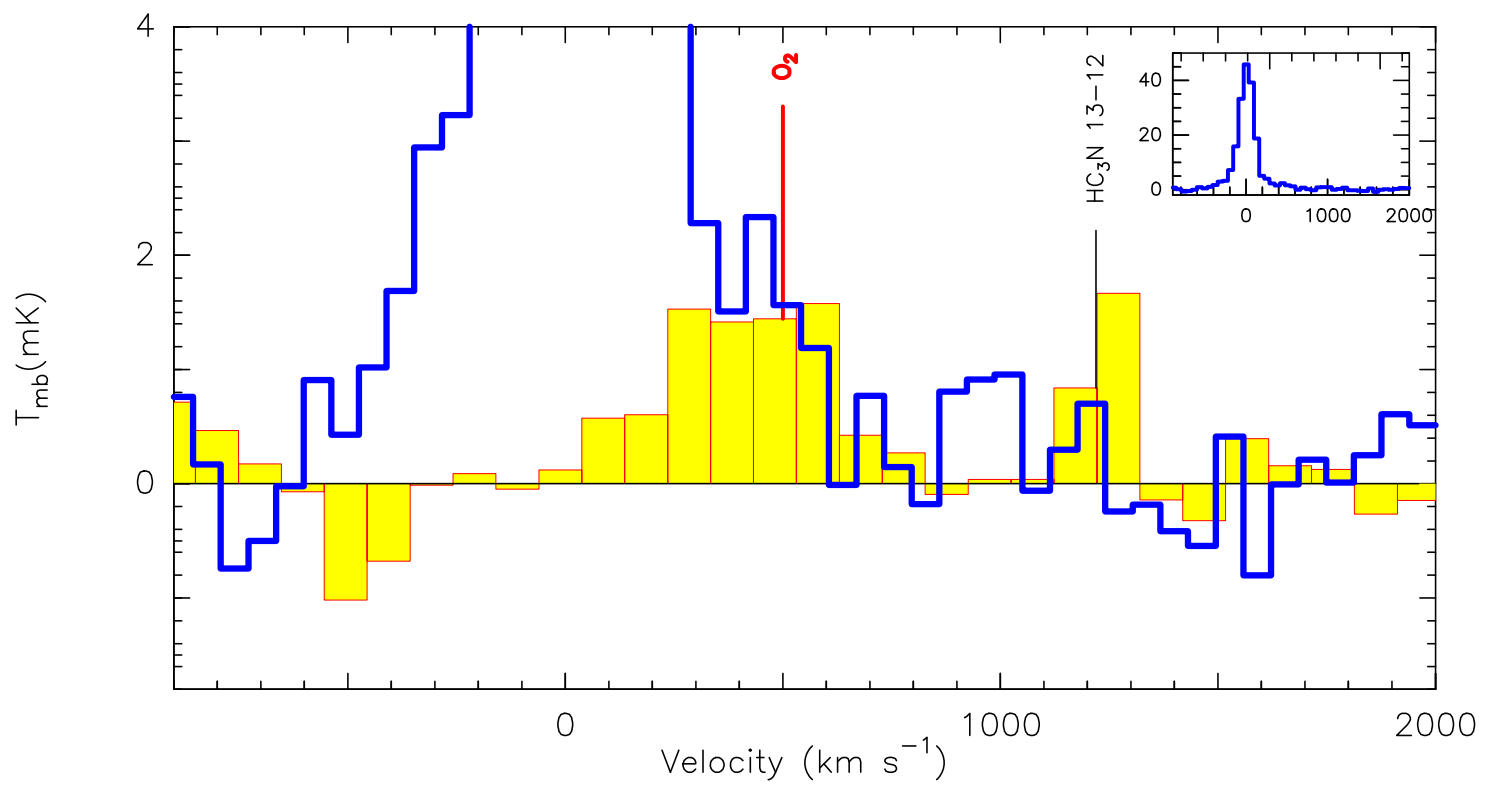

Figure 1. $\mathrm{O}_{2} N_{J}=1_{1}-1_{0}$ line emission having rest frequency $118.75034 \mathrm{GHz}$ (filled yellow histogram and red line) in Mrk 231 observed with the IRAM $30 \mathrm{~m}$ telescope. The spectrum combines all data obtained in 4 days. The $x$-axis is radio-defined velocity, which is referred to the frequency of redshifted $\mathrm{O}_{2}$ as $118.75034 /$ $(1+z) \mathrm{GHz}$ with $z=0.04217$. The velocity resolution presented here has been smoothed to $98.66 \mathrm{~km} \mathrm{~s}^{-1}$. The blue line is the CO $1-0$ data obtained simultaneously, aligned with radio-defined velocity referred to the frequency of redshifted CO $1-0$ as $115.271202 /(1+z) \mathrm{GHz}$. The $\mathrm{HC}_{3} \mathrm{~N} J=13-12$ line having rest frequency of $118.2707 \mathrm{GHz}$ was observed with and is plotted on the same velocity scale as the $\mathrm{O}_{2}$. It appears at a velocity $\simeq 1200 \mathrm{~km} \mathrm{~s}^{-1}$ as the emission velocity of this dense gas tracer is close to $0 \mathrm{~km} \mathrm{~s}^{-1}$, the systemic velocity of Mrk 321. The $y$-axis is the main beam temperature in millikelvin for both $\mathrm{O}_{2}$ and $\mathrm{CO} 1-0$ spectra. The spectrum in the insert box (blue line) gives a view of the complete CO line over the same velocity range as in the main figure.

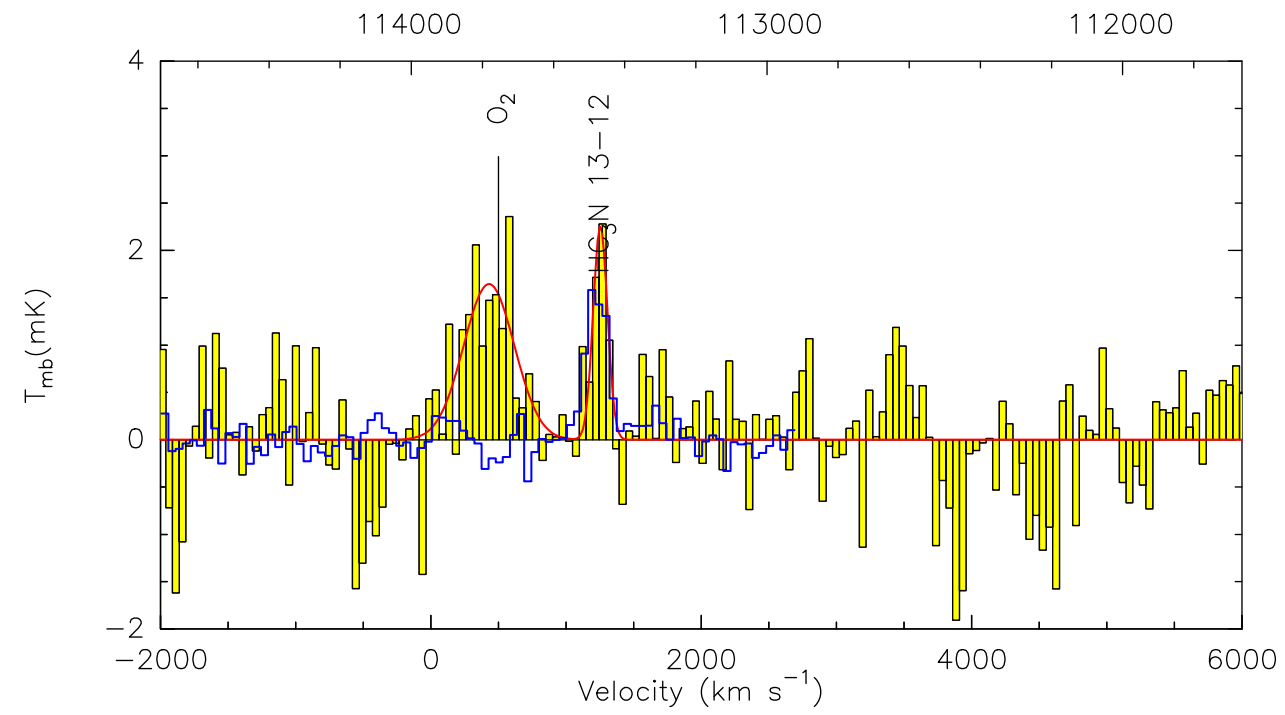

Figure 2. Spectrum (black and filled yellow) in Mrk 231 with the same data shown in Figure 1 presented with less velocity smoothing and broader velocity coverage. The $x$-axis is velocity as defined in Figure 1 . The rms of this spectrum is $0.65 \mathrm{mK}$ at a frequency resolution of $18.75 \mathrm{MHz}$, corresponding to velocity resolution $49.33 \mathrm{~km} \mathrm{~s}^{-1}$. The red line is the result of two-component Gaussian fitting. The velocity coverage is from -2000 to $6000 \mathrm{~km} \mathrm{~s}^{-1}$, which represents about $3000 \mathrm{MHz}$ frequency coverage as indicated by the frequency scale (in MHz) at the top of the figure. The overlaid blue spectrum is from the central region of Mrk231 obtained with NOEMA, which is the same as that in the upper left in Figure 4.

is $1.9 \pm 0.2 \mathrm{Jy} \mathrm{km} \mathrm{s}^{-1}$, which includes about $43 \%$ of the emission detected with the $30 \mathrm{~m}$ telescope, and covers a consistent velocity range, from about 180 to $570 \mathrm{~km} \mathrm{~s}^{-1}$. Since the signal is not a point source located at the phase center, which leads to the difficulty of obtaining the emission features in UV plane data, the UV spectrum was not presented.

Continuum subtraction was implemented in the cleaned image data cube based on nearby line free channels, from about -2500 to $-1000 \mathrm{~km} \mathrm{~s}^{-1}$ and from about 1600 to $3000 \mathrm{~km} \mathrm{~s}^{-1}$. $\mathrm{HC}_{3} \mathrm{~N}$ 13-12 was seen as compact emission in the nuclear region, coincident with the AGN/extreme starburst and consistent with the spectrum taken by the IRAM $30 \mathrm{~m}$ telescope, while $\mathrm{O}_{2}$ emission was observed to be extended in outer disk regions $\sim 10 \mathrm{kpc}$ from the nucleus. The emission was detected in several different regions with different velocities (see Figures 4 and 5). The flux of the $\mathrm{HC}_{3} \mathrm{~N}$ 13-12 line detected with NOEMA is $1.55 \pm 0.07 \mathrm{Jy} \mathrm{km} \mathrm{s}^{-1}$, which agrees with the total emission detected with the IRAM $30 \mathrm{~m}$ single-dish telescope within the uncertainties of the observations at the two facilities.

We present the velocity-integrated maps for different velocity ranges in Figure 5, smoothed to about $150 \mathrm{~km} \mathrm{~s}^{-1}$ velocity resolution. The noise level $(1 \sigma)$ is about $0.045 \mathrm{Jy} \mathrm{km} \mathrm{s}^{-1}$ for 

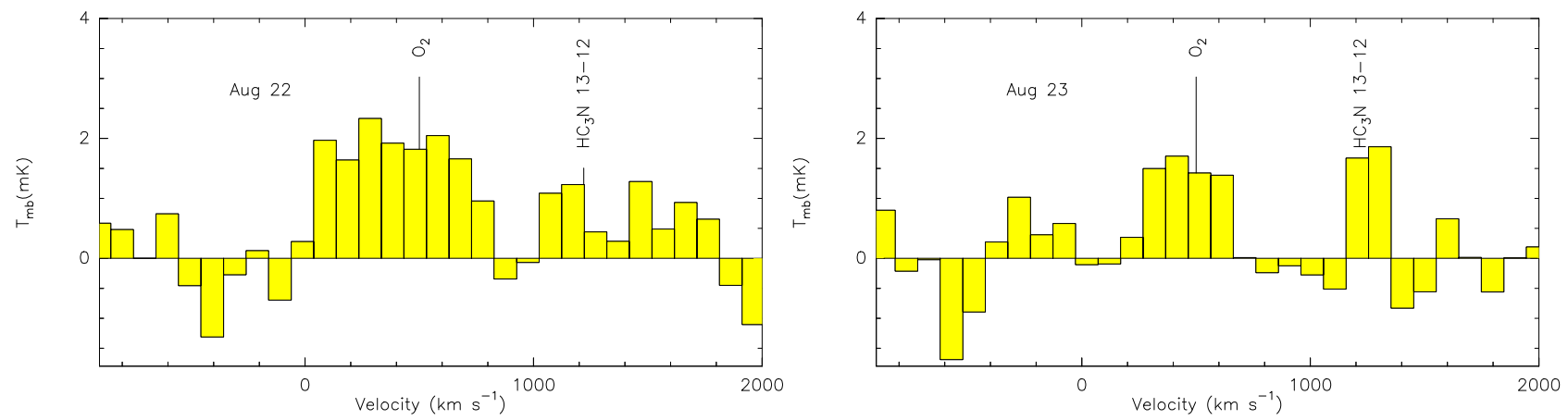

Figure 3. Spectra in Mrk 231 observed with the IRAM $30 \mathrm{~m}$ telescope on 2015 August 22 (left), and August 23 (right).
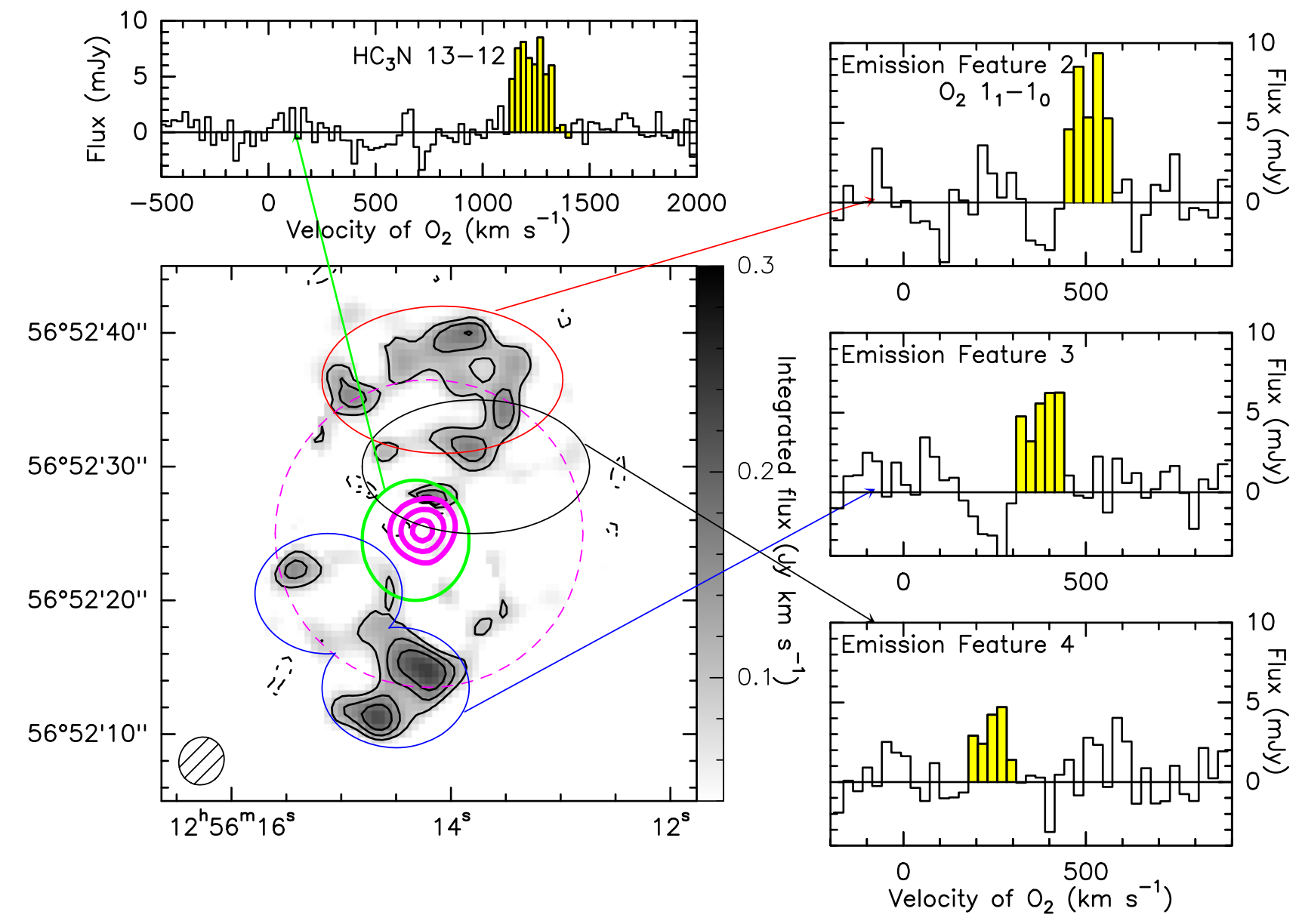

Figure 4. $\mathrm{O}_{2}$ emission detected with the IRAM NOEMA interferometer. The gray scale and contours in black are the velocity-integrated $\mathrm{O}_{2}$ emission in units of $\mathrm{Jy} \mathrm{km} \mathrm{s}{ }^{-1}$, integrated over $180-570 \mathrm{~km} \mathrm{~s}^{-1}$. The grayscale colorbar at the right is in units of Jy km s${ }^{-1}$. The contours are from $2 \sigma$ increasing in steps of $1 \sigma$, which corresponds to $0.045 \mathrm{Jy} \mathrm{km} \mathrm{s}^{-1}$. The contours in magenta are the velocity-integrated $\mathrm{HC}_{3} \mathrm{~N} 13-12$ emission starting from and with steps of $0.4 \mathrm{Jy} \mathrm{km} \mathrm{s}{ }^{-1}$. The ellipses are the regions over which data were integrated to obtain the spectra, including that for $\mathrm{HC}_{3} \mathrm{~N}$ 13-12 emission, with the same definition of velocity as in Figure 1. The

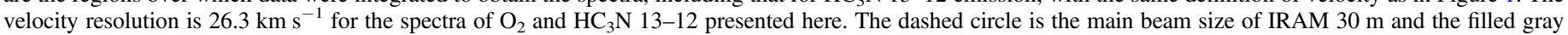
ellipse at bottom left is the beam size of NOEMA data $\left(3.63^{\prime \prime} \times 3.32^{\prime \prime}, \mathrm{PA}=-65^{\circ}\right)$.

each map, while the contours are from $2 \sigma$ with $1 \sigma$ steps. Emission features can be seen in several velocity components above $3 \sigma$, especially from 180 to $570 \mathrm{~km} \mathrm{~s}^{-1}$, which is consistent with the emission feature detected with the IRAM $30 \mathrm{~m}$ telescope. The $\mathrm{O}_{2}$ moment zero map (Figure 4, in both gray scale and contours) includes emission features in the velocity range $180-570 \mathrm{~km} \mathrm{~s}^{-1}$. The corresponding angular regions are marked in Figure 5. With information from NOEMA observations, even the velocity range of $\mathrm{O}_{2} N_{J}=1_{1}-1_{0}$ emission is similar to that of $\mathrm{CO}$ red wing, which traces molecular outflows within 3" (Feruglio et al. 2010; Cicone et al. 2012), $\mathrm{O}_{2}$ is from different regions of outflowing gas traced by $\mathrm{CO}$ red wing.

\subsection{Line Identification as $O_{2} \mathrm{~N}_{\mathrm{J}}=1_{1}-1_{0}$ Emission}

The image rejection of the EMIR on the IRAM $30 \mathrm{~m}$ telescope is better than $13 \mathrm{~dB}$, which corresponds to $\geqslant 20: 1$. The lower sideband image frequency corresponding to the feature of interest in the upper sideband is different on different 
Table 1

Emission Features of $\mathrm{O}_{2} N_{J}=1_{1}-1_{0}$ Detected with the IRAM $30 \mathrm{~m}$ and NOEMA

\begin{tabular}{lcccl}
\hline \hline $\begin{array}{l}\text { Emission } \\
\text { Feature }\end{array}$ & $\begin{array}{c}\text { Flux } \\
\left(\mathrm{Jy} \mathrm{km} \mathrm{s}^{-1}\right)\end{array}$ & $\begin{array}{c}\text { Line Center } \\
\left(\mathrm{km} \mathrm{s}^{-1}\right)\end{array}$ & $\begin{array}{c}\text { Line Width } \\
\left(\mathrm{km} \mathrm{s}^{-1}\right)\end{array}$ & Telescope \\
\hline 1 & $3.7 \pm 0.3$ & $418.7 \pm 47.9$ & $456.0 \pm 107.1$ & $30 \mathrm{~m}$ \\
2 & $0.80 \pm 0.13$ & $511.5 \pm 8.1$ & $93.5 \pm 13.1$ & NOEMA \\
3 & $0.70 \pm 0.12$ & $391.5 \pm 9.7$ & $98.8 \pm 16.7$ & NOEMA \\
4 & $0.36 \pm 0.10$ & $248.5 \pm 11.9$ & $77.6 \pm 24.7$ & NOEMA
\end{tabular}

Note. The errors only include the deviation from Gaussian fitting. About $10 \%$ $20 \%$ of the velocity-integrated flux should be also considered as the uncertainty for comparison with other observations, arising from the uncertainties in the absolute flux calibration and pointing errors for the single-dish observations. Emission features 2-4 correspond to the three spectra in the right of Figure 4, while emission feature 1 corresponds to the spectrum in Figures 1 and 2 .

days, since different LO frequencies were used. The result is that any signal from the lower sideband will move around in frequency rather than adding together when the data from different days are added. The image frequencies are in the range 96-97.5 GHz during the four days, and are not near the strong dense gas tracer CS $J=2-1$ at the rest frequency of $97.980953 \mathrm{GHz}$. Even the strongest dense gas tracer in the $3 \mathrm{~mm}$ band, $\mathrm{HCN} 1-0$, produced an antenna temperature of only $6.5 \mathrm{mK}$ in Mrk 231 with the IRAM $30 \mathrm{~m}$ telescope (Jiang et al. 2011). The lower sideband image frequencies with NOEMA corresponding to the upper sideband emission feature identified as $\mathrm{O}_{2} N_{J}=1_{1}-1_{0}$ are between 92 and $94 \mathrm{GHz}$ (due to the different IF frequency), and there were no emission features detected in the lower sideband, while the detected CS $J=2-1$ line with NOEMA observation is about half the intensity of $\mathrm{HCN} J=1-0$. The expected confusion from the lines in the image sideband should thus be much less than $0.2 \mathrm{mK}$ for the final spectrum, which does not affect the line identification.

The rest frequency range of the emission feature from -100 to $900 \mathrm{~km} \mathrm{~s}^{-1}$ in Figure 1 is from about 118.4 to $118.8 \mathrm{GHz}$. From splatalogue (http://www.cv.nrao.edu/php/splat/), which combines the JPL molecular catalog (Pickett et al. 1998) and the CDMS catalog (Müller et al. 2001), there are 350 lines in this frequency range, including the $\mathrm{O}_{2} N_{J}=1_{1}-1_{0}$ line at $118.75034 \mathrm{GHz}$, which is the only one that has been detected in space (Larsson et al. 2007). With the criterion of lower level energy less than $100 \mathrm{~K}$, the number of lines is decreased to 83, which are mainly from complex molecules, such as $\mathrm{CH}_{3} \mathrm{OCH}_{3}$ and CCCN, or isotopologues, such as ${ }^{33} \mathrm{SO}_{2}$. The abundances of such complex molecules and isotopologues are not expected to be high enough to produce emission stronger than $\mathrm{HC}_{3} \mathrm{~N}$ $J=13-12$ in Mrk 231. Otherwise, such features should be easily detected in Galactic molecular clouds, while $\mathrm{HC}_{3} \mathrm{~N} J=13-12$ was the strongest (and possibly the only) emission line detected in the frequency range from $117.8 \mathrm{GHz}$ to $118.75 \mathrm{GHz}$ toward the Sgr A complex region in the Galactic center with Odin (Sandqvist et al. 2008).

If we restrict the emission feature to fall within $\pm 100 \mathrm{~km} \mathrm{~s}^{-1}$ of the central velocity of the galaxy, with the rest frequency range from 118.54 to $118.62 \mathrm{GHz}$, there are 57 lines from splatalogue, none of which has been reported as detected. All of them, except for $\mathrm{SO}_{2}$ at $118.57743 \mathrm{GHz}$ and the vibrationally excited $\mathrm{HC}_{3} \mathrm{~N} J=13-12$ e line at $118.56145 \mathrm{GHz}$, are extremely complex molecules with more than five atoms. The lower level energy of $\mathrm{SO}_{2}$ and $\mathrm{HC}_{3} \mathrm{~N}$ transitions are from about 250 to $1600 \mathrm{~K}$, which makes them unlikely candidates for the observed emission. In addition, the vibrationally excited line would almost certainly be significantly weaker than the corresponding ground vibrational state line, as found even in regions of high infrared flux that can radiatively excite the vibrational levels, e.g., Goldsmith et al. (1982).

If the origin of the emission were a low-lying transition of a complex molecule, it should have been detected in sources in the Milky Way. However, no such emission feature was reported by Sandqvist et al. (2008). Thus, the most probable identification of the emission feature from 100 to $800 \mathrm{~km} \mathrm{~s}^{-1}$ is $\mathrm{O}_{2} N_{J}=1_{1}-1_{0}$ with the velocity of the red wing of the gas in Mrk 231 .

\section{Discussion}

\section{1. $\mathrm{O}_{2}$ Abundance in Different Regions of Mrk 231: Outer Disk versus Central $2 \mathrm{kpc}$}

Stellar components traced at optical wavelengths can be seen (Rupke \& Veilleux 2011) in the region with high velocity redshifted $\mathrm{O}_{2}$ emission, while studies of molecular gas traced by CO lines (Feruglio et al. 2010; Cicone et al. 2012; Feruglio et al. 2015) were focused on the central $2-3 \mathrm{kpc}$ region, even though some outflow features can be seen up to $5^{\prime \prime}(\sim 4 \mathrm{kpc})$ north of the central region (Cicone et al. 2012). In the region with $\mathrm{O}_{2}$ detection extending from 450 to $570 \mathrm{~km} \mathrm{~s}^{-1}$, the flux of the simultaneously obtained $\mathrm{CO} J=1-0$ line was about four times that of the $\mathrm{O}_{2} N_{J}=1_{1}-1_{0}$ line. However, the velocity range of $\mathrm{CO} 1-0$ is primarily associated with the central region of Mrk 231, which is quite distinct from the $\mathrm{O}_{2}$ emission. Thus, even though the $\mathrm{O}_{2}$ emission has a velocity range similar to the red wing of molecular outflow traced by $\mathrm{CO} 1-0$, it is coming from a different portion of Mrk 231. Molecular line emission of $\mathrm{CH}^{+} J=1-0$ without associated $\mathrm{CO}$ emission has been found in several high redshift starburst galaxies (Falgarone et al. 2017), which indicates that CO emission is not necessarily detectable in the regions in which some special molecules, such as $\mathrm{O}_{2}$ and $\mathrm{CH}^{+}$can be detected.

The CO $J=1-0$ fluxes in the regions with $\mathrm{O}_{2}$ detections were comparable to, or even less than that of $\mathrm{O}_{2} N_{J}=1_{1}-1_{0}$ line, if we consider that only the $\mathrm{CO} 1-0$ emission with the same velocity range as the $\mathrm{O}_{2}$ line is from the same gas as responsible for the $\mathrm{O}_{2}$ emission. Using the same $\mathrm{CO} 1-0$ flux to $\mathrm{H}_{2}$ conversion factor in the $\mathrm{O}_{2}$ emission region as that of the host galaxy and assuming that the $\mathrm{O}_{2}$ line is optically thin with a $15 \mathrm{~K}$ excitation temperature $\left(T_{\mathrm{ex}}\right)$, which is the same as was assumed in NGC 6240 (Combes et al. 1991), the $\mathrm{O}_{2}-\mathrm{H}_{2}$ abundance ratio can be estimated to be higher than $10^{-4}$ (see Table 2), nearly two orders of magnitude higher than that of Galactic sources detected in $\mathrm{O}_{2}$, and higher than the CO-to- $\mathrm{H}_{2}$ abundance ratio. The dependence of the $\mathrm{O}_{2}$ column density on its excitation temperature in LTE is $\mathrm{N}\left(\mathrm{O}_{2}\right) \propto T_{\mathrm{ex}}^{0.67}$ (Liseau \& $\mathrm{O}_{2}$ din Team 2005). Thus if the excitation temperature of $\mathrm{O}_{2}$ were higher than $15 \mathrm{~K}$, the $\mathrm{O}_{2}-\mathrm{H}_{2}$ abundance ratio would be even higher.

The nondetection of $\mathrm{O}_{2}$ emission in the central $2 \mathrm{kpc}$ of Mrk 231 with our NOEMA observations gave the best upper limit at the extragalactic $\mathrm{O}_{2} \quad N_{J}=1_{1}-1_{0}$ to $\mathrm{CO} 1-0$ line ratio of $1 \times 10^{-3}(1 \sigma)$, much lower than the $1.2 \times 10^{-2}$ value for NGC 6240, which was used previously for estimating the $\mathrm{O}_{2} / \mathrm{H}_{2}$ abundance ratio (Combes et al. 1991). Using the same 


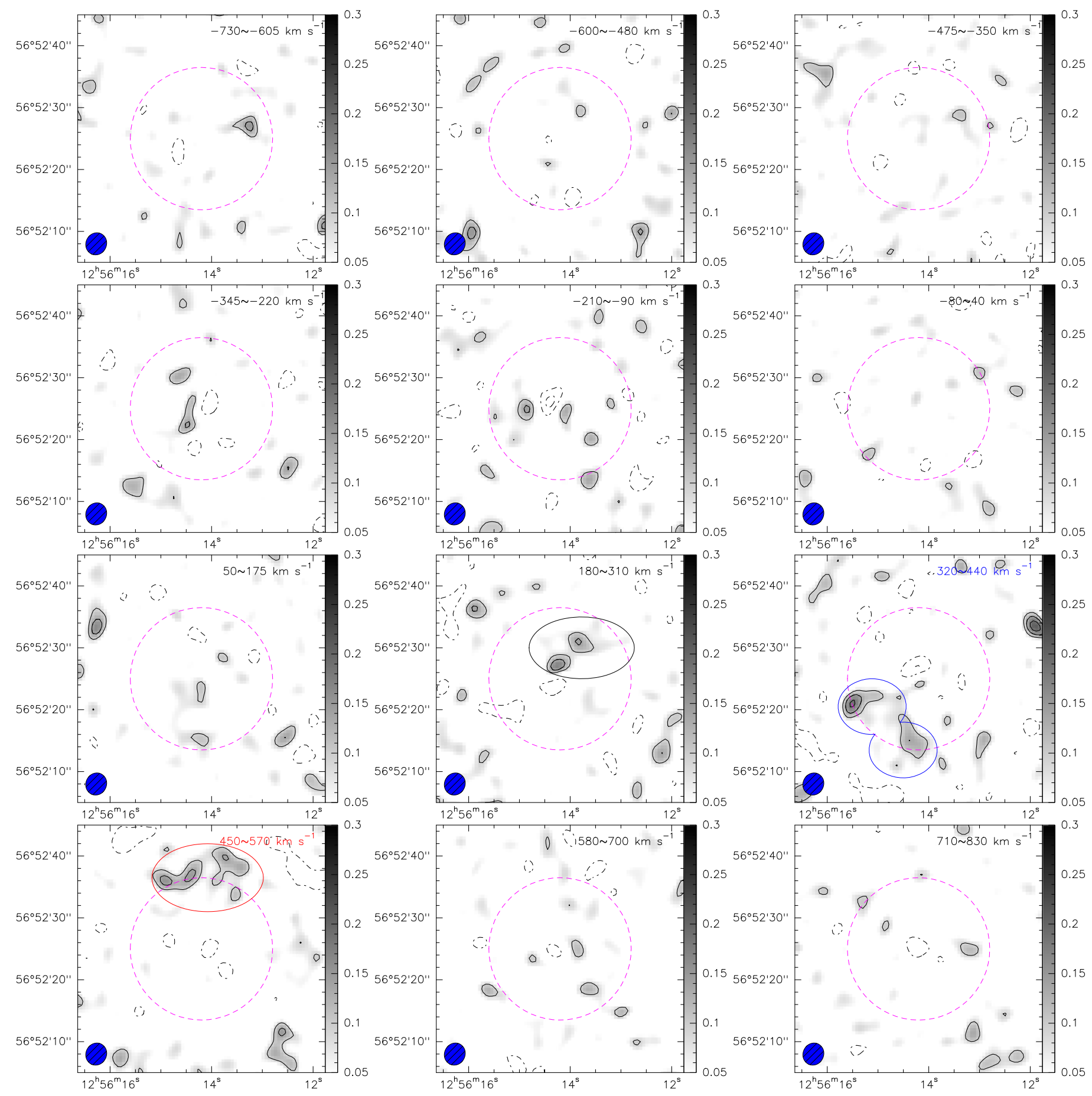

Figure 5. Velocity-integrated maps of $\mathrm{O}_{2}$ emission for different velocity ranges with the IRAM NOEMA data. The contours are from $2 \sigma$ increasing in steps of $1 \sigma$, which corresponds to $0.045 \mathrm{Jy} \mathrm{km} \mathrm{s}^{-1}$. The color bars, circles, and ellipses are the same as in Figure 4.

$\mathrm{CO} J=1-0$ flux to $\mathrm{H}_{2}$ conversion factor as that in NGC 6240 and similar excitation conditions of the $\mathrm{O}_{2}$ molecules (Combes et al. 1991), the $\mathrm{O}_{2}$ to $\mathrm{H}_{2}$ abundance ratio should be less than $8 \times 10^{-8}$ in the host galaxy of Mrk 231, which is consistent with that found in the vast majority of dense molecular gas in the Milky Way (Goldsmith et al. 2000; Pagani et al. 2003). The only exceptions are Orion (Goldsmith et al. 2011) (having significantly higher abundance in $10^{-6}$ in a very small region) and $\rho$ Oph (Larsson et al. 2007; Liseau et al. 2012) (with comparable abundance $5 \times 10^{-8}$ ).
We compiled the sources with $\mathrm{O}_{2}$ detections, the best upper limit of $\mathrm{O}_{2}-\mathrm{H}_{2}$ abundance ratio in NGC 6240 with emission (Combes et al. 1991) and in B0218+357 with absorption (Combes et al. 1997) in the literature, and show the results in Table 2. If we wish to detect $\mathrm{O}_{2} N_{J}=1_{1}-1_{0}$ emission in extragalactic sources without $\mathrm{O}_{2}$ abundance enhancement, observations of the redshifted $\mathrm{O}_{2} N_{J}=1_{1}-1_{0}$ line with five times lower noise level than that of our observations with NOEMA toward Mrk 231 are needed. With the Atacama Large Millimeter/submillimeter array (ALMA), about 10 hours 
Table 2

$\mathrm{O}_{2}$ Detections and Best Upper Limits

\begin{tabular}{|c|c|c|c|}
\hline Source & $\mathrm{O}_{2}-\mathrm{H}_{2}$ abundance ratio & Telescope & References \\
\hline Orion & $1 \times 10^{-6}$ & Herschel & Goldsmith et al. (2011) \\
\hline$\rho \mathrm{Oph}$ & $5 \times 10^{-8}$ & Odin & Larsson et al. (2007) \\
\hline NGC 6240 & $<1 \times 10^{-6}$ & IRAM $30 \mathrm{~m}$ & Combes et al. (1991) \\
\hline $\mathrm{B} 0218+357$ & $<2 \times 10^{-7}$ & NRAO $12 \mathrm{~m}$ & Combes et al. (1997) \\
\hline Mrk231 (central 2kpc) & $<8 \times 10^{-8}$ & IRAM NOEMA & This work \\
\hline Mrk231 (outflow) & $>1 \times 10^{-4}$ & IRAM $30 \mathrm{~m}$ and NOEMA & This work \\
\hline
\end{tabular}

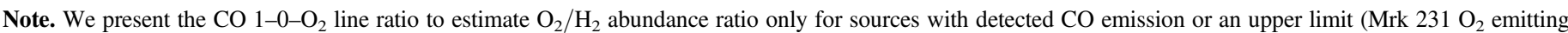

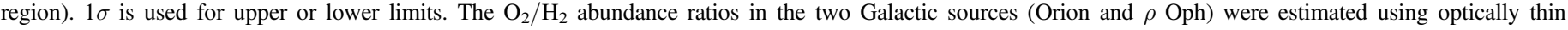
isotopologues instead of $\mathrm{CO}$, while the ratio for $\mathrm{B} 0218+357$ was estimated based on an absorption spectrum.

on-source time toward nearby gas-rich galaxies with bright $\mathrm{CO}$ emission can achieve such sensitivity.

\subsection{Origin of Molecular Oxygen: Large-Scale Shock Caused by AGN Molecular Outflow?}

The velocity range of our $\mathrm{O}_{2}$ emission is mainly from redshifted gas, especially from $\sim 345$ to $615 \mathrm{~km} \mathrm{~s}^{-1}$, with projected distance of about $10 \mathrm{kpc}$ north and south from the central region, while the low velocity components, from about -400 to $+300 \mathrm{~km} \mathrm{~s}^{-1}$, were seen as weaker emission and at smaller projected distances to the center (see Figure 4). On the other hand, high velocity blueshifted components were even weaker than the low velocity ones and were hardly detected in this observation. The asymmetry in spatial and velocity distribution of $\mathrm{O}_{2}$ emission, including the absence of $\mathrm{O}_{2}$ emission along the line of sight around the nuclear region, can be attributed to the geometry of molecular gas in Mrk 231 and the asymmetry of the molecular outflow from the central AGN, which had been found in $\mathrm{CO}$ observations up to about $3 \mathrm{kpc}$ scale away from the center of Mrk 231 in projection (Feruglio et al. 2010; Cicone et al. 2012).

The absence of a blueshifted component of $\mathrm{O}_{2}$ emission may be caused by asymmetric distribution of molecular gas in the regions about $10 \mathrm{kpc}$ away from the center, because $\mathrm{O}_{2}$ should be produced by the interaction between the outflowing gas from the AGN center and the local molecular gas there. With velocity of about $500 \mathrm{~km} \mathrm{~s}^{-1}, 20 \mathrm{Myr}$ are required for gas to travel $10 \mathrm{kpc}$, which is the typical projected distance between the $\mathrm{O}_{2}$ emission regions and the center of Mrk 231. Thus $\mathrm{O}_{2}$ emission in Mrk 231 traces the outflow from the central AGN on a characteristic dynamic timescale of $20 \mathrm{Myr}$.

X-ray emission extended up to $25 \mathrm{kpc}$ away from the center of Mrk 231 was found with half-mega second Chandra spectral imaging. A merger remnant was proposed as the main contributor to the large-scale X-ray halo of Mrk 231 (Veilleux et al. 2014). However, the central AGN is responsible for emission at smaller ( $\leqslant 6 \mathrm{kpc})$ distances. We suggest that large scale AGN molecular outflow is the most plausible mechanism to produce a shock that drives the enhancement of the $\mathrm{O}_{2}$ abundance but a merger is another possibility for producing a shock (or multiple shocks) that enhanced the abundance of $\mathrm{O}_{2}$.

Models of shock chemistry (Chen et al. 2014; Neufeld et al. 2014; Melnick \& Kaufman 2015; Godard et al. 2019) suggest that essentially all of the gas-phase oxygen will be incorporated into $\mathrm{H}_{2} \mathrm{O}$ and $\mathrm{CO}$ if the shock velocity exceeds $20 \mathrm{~km} \mathrm{~s}^{-1}$. Thus, a single shock that provides the velocity offset of the redshifted $\mathrm{O}_{2}$ emission of Mrk 231 could not be responsible for dramatically enhancing the abundance of molecular oxygen.
Rather, a number of lower velocity shocks produced in the outflow, having different velocities relative to the general outflow, could collectively provide the observed large $\mathrm{O}_{2}$ abundance. A high $\mathrm{O} / \mathrm{CO}$ ratio up to $2 \times 10^{4}$ can be produced in a model of a UV irradiated molecular shock (Godard et al. 2019). Such irradiated molecular clouds with shocks located far away from the center of galaxies can have physical properties similar to those of the $\mathrm{O}_{2}$-emitting regions in Mrk 231 . Chemical networks coupled with dynamic models including radiation field are necessary for understanding the high $\mathrm{O}_{2} / \mathrm{CO}$ abundance ratio there.

For $15 \mathrm{~K}$ gas under LTE conditions, the $118.75034 \mathrm{GHz} \mathrm{O}_{2}$ transition is the brightest among the $114 \mathrm{O}_{2}$ lines listed between 10 and $1000 \mathrm{GHz}$ (Pickett et al. 1998). In total, only 12 transitions make meaningful contributions, which is consistent with what had been predicted with an LVG model (Liseau \& $\mathrm{O}_{2}$ din Team 2005). The total $\mathrm{O}_{2}$ flux amounts to 2.9 times the flux at $118 \mathrm{GHz}$. Similar procedures applied to 17 transitions of $\mathrm{CO}$ yielded a $\mathrm{CO}$ total flux 5.1 times that of $\mathrm{CO} 1-0$. Given the comparable fluxes of the $\mathrm{O}_{2} N_{J}=1_{1}-1_{0}$ transition and the CO 1-0 line in outer disk region of Mrk 231, the $\mathrm{O}_{2}$ molecule is comparable to $\mathrm{CO}$ as an important coolant of such molecular gas.

Based on CO observations (Cicone et al. 2014; Fluetsch et al. 2019), massive molecular outflows, which have negative feedback on star formation in galaxies, are quite common in AGN. The $\mathrm{O}_{2} N_{J}=1_{1}-1_{0}$ transition is thus a promising new tool for studying such outflows on dynamic timescales of a few tens of megayears. AGNs with molecular gas detected via strong $\mathrm{CO}$ emission will be good candidates for searching for $\mathrm{O}_{2}$ emission in extragalactic sources.

\section{Summary and Future Prospects}

With deep observations toward Mrk 231 using the IRAM $30 \mathrm{~m}$ telescope and NOEMA, we detected $\mathrm{O}_{2} N_{J}=1_{1}-1_{0}$ emission in external galaxy for the first time, at about the $12 \sigma$ level. The detected $\mathrm{O}_{2}$ emission is located in regions about $10 \mathrm{kpc}$ away from the center of Mrk 231 and may be caused by the interaction between the AGN-driven molecular outflow and the outer disk molecular clouds.

Massive molecular outflows are quite common in AGN based on $\mathrm{CO}$ observations (Cicone et al. 2014). The $\mathrm{O}_{2}$ $N_{J}=1_{1}-1_{0}$ transition could prove to be a new tool for studying the effect of such outflows, because outflows identified with $\mathrm{CO}$ observations can be severely contaminated by emission components of the host galaxy. On the other hand, $\mathrm{O}_{2}$ emission from dense molecular gas in the host galaxy can be neglected, which means $\mathrm{O}_{2}$ emission can be used to study a molecular 
outflow even if it is perpendicular to the line of sight. AGNs with molecular outflows detected in $\mathrm{CO}$ emission are good candidates for detecting $\mathrm{O}_{2}$ emission in extragalactic sources. Even though Mrk 231, a northern source at decl. of $\sim 57^{\circ}$, cannot be reached with ALMA, other redshifted AGNs with molecular outflows may be studied with high resolution high sensitivity $\mathrm{O}_{2} \quad N_{J}=1_{1}-1_{0}$ observations using ALMA and NOEMA, as well as the Next-Generation Very Large Array (ngVLA) in the next decade. $\mathrm{O}_{2}$ lines at submillimeter wavelengths, such as that at $424.76 \mathrm{GHz}$, with suitable redshift shifting it to an observable window of ALMA, may be useful for studying molecular oxygen in more distant galaxies.

This work is supported by the National Natural Science Foundation of China grants 11988101, 11590783, 11988101, 11590782, 11690024, and 11725313 and the International Partnership Program of Chinese Academy of Sciences grant No. 114A11KYSB20160008. This study is based on observations carried out under project number 068-15 with the IRAM $30 \mathrm{~m}$ telescope and d17ae001 with NOEMA. IRAM is supported by INSU/CNRS (France), MPG (Germany), and IGN (Spain). This research was carried out in part at the Jet Propulsion Laboratory which is operated for NASA by the California Institute of Technology. Y.G.'s research is supported by the National Key Basic Research and Development Program of China (grant No. 2017YFA0402704), the National Natural Science Foundation of China (grant Nos. 11861131007, 11420101002), and the Chinese Academy of Sciences Key Research Program of Frontier Sciences (grant No. QYZDJSSWSLH008). This work also benefited from the International Space Science Institute (ISSI/ISSI-BJ) in Bern and Beijing, thanks to the funding of the team "Chemical abundances in the ISM: the litmus test of stellar IMF variations in galaxies across cosmic time" (Principal Investigators D.R. and Z.-Y.Z.). We thank the anonymous referee for helpful comments, which improved the manuscript.

\section{ORCID iDs}

Junzhi Wang (D) https://orcid.org/0000-0001-6106-1171

Di Li ib https://orcid.org/0000-0003-3010-7661

Paul F. Goldsmith (iD https://orcid.org/0000-0002-6622-8396
Zhi-Yu Zhang (iD https://orcid.org/0000-0002-7299-2876

Yu Gao (i) https://orcid.org/0000-0003-0007-2197

Yong Shi (1) https://orcid.org/0000-0002-8614-6275

Min Fang (1D https://orcid.org/0000-0001-8060-1321

Jiangshui Zhang (1) https://orcid.org/0000-0002-5161-8180

\section{References}

Aalto, S., Garcia-Burillo, S., Muller, S., et al. 2012, A\&A, 537, A44 Bergin, E. A., Melnick, G. J., \& Neufeld, D. A. 1998, ApJ, 499, 777 Bryant, P. M., \& Scoville, N. Z. 1996, ApJ, 457, 678

Carilli, C. L., Wrobel, J. M., \& Ulvestad, J. S. 1998, AJ, 115, 928

Chen, J.-H., Goldsmith, P. F., Viti, S., et al. 2014, ApJ, 793, 111

Cicone, C., Feruglio, C., Maiolino, R., et al. 2012, A\&A, 543, A99

Cicone, C., Maiolino, R., Sturm, E., et al. 2014, A\&A, 562, A21

Combes, F., Casoli, F., Encrenaz, P., et al. 1991, A\&A, 248, 607

Combes, F., Wiklind, T., \& Nakai, N. 1997, A\&A, 327, L17

Falgarone, E., Zwaan, M. A., Godard, B., et al. 2017, Natur, 548, 430

Feruglio, C., Fiore, F., Carniani, S., et al. 2015, A\&A, 583, A99

Feruglio, C., Maiolino, R., Piconcelli, E., et al. 2010, A\&A, 518, L155

Fischer, J., Sturm, E., González-Alfonso, E., et al. 2010, A\&A, 516, L41

Fluetsch, A., Maiolino, R., Carniani, S., et al. 2019, MNRAS, 483, 4586

Frayer, D. T., Seaquist, E. R., Thuan, T. X., \& Sievers, A. 1998, ApJ, 503, 231

Godard, B., Pineau des Forêts, G., Lesaffre, P., et al. 2019, A\&A, 622, A100

Goldsmith, P. F., Li, D., Bergin, E. A., et al. 2002, ApJ, 576, 814

Goldsmith, P. F., Liseau, R., Bell, T. A., et al. 2011, ApJ, 737, 96

Goldsmith, P. F., Melnick, G. J., Bergin, E. A., et al. 2000, ApJL, 539, 123

Goldsmith, P. F., Snell, R. L., Deguchi, S., et al. 1982, ApJ, 260, 147

Goldsmith, P. F., \& Young, J. S. 1989, ApJ, 341, 718

Hollenbach, D., Kaufman, M. J., Bergin, E. A., \& Melnick, G. J. 2009, ApJ, 690,1497

Jiang, X., Wang, J., \& Gu, Q. 2011, MNRAS, 418, 1753

Kanekar, N., \& Meier, D. S. 2015, ApJL, 811, 23

Langer, W. D., \& Graedel, T. E. 1989, ApJS, 69, 241

Larsson, B., Liseau, R., Pagani, L., et al. 2007, A\&A, 466, 999

Liseau, R. \& $\mathrm{O}_{2}$ din Team 2005, in IAU Symp. 231, Recent Successes and Current Challenges, ed. D. C. Lis, G. A. Blake, \& E. Herbst (Cambridge: Cambridge Univ. Press), 301

Liseau, R., Goldsmith, P. F., Larsson, B., et al. 2012, A\&A, 514, A73

Liszt, H. S. 1985, ApJ, 298, 281

Melnick, G. J., \& Kaufman, M. J. 2015, ApJ, 806, 227

Müller, H. S. P., Thorwirth, S., Roth, D. A., \& Winnewisser, G. 2001, A\&A, 370, L49

Neufeld, D. A., Gusdorf, A., Güsten, R., et al. 2014, ApJ, 781, 102

Pagani, L., Olofsson, A. O. H., Bergman, P., et al. 2003, A\&A, 402, L77

Pickett, H. M., Poynter, R. L., Cohen, E. A., et al. 1998, JQSRT, 60, 883

Rupke, D. S. N., \& Veilleux, S. 2011, ApJL, 729, L27

Sandqvist, A., Larsson, B., Hjalmarson, A, et al. 2008, MNRAS, 482, 849

Veilleux, S., Teng, S. H., Rupke, D. S. N., et al. 2014, ApJ, 790, 116 